\title{
Optimization and validation of the protocol used to analyze the taste of traditional Chinese medicines using an electronic tongue
}

\author{
XUELIN LI ${ }^{1 *}$, XIAOJIE GAO ${ }^{2 *}$, RUIXIN LIU ${ }^{1,3,4}$, JUNMING WANG ${ }^{2}$, ZIDAN WU $^{2,5}$, \\ LU ZHANG ${ }^{1,3,4}$, HUILING LI ${ }^{2}$, XINJING GUI ${ }^{2}$, BINGYA KANG ${ }^{1,3,4}$ and JUNHAN SHI ${ }^{1,3,4}$
}

\begin{abstract}
${ }^{1}$ Department of Pharmacy, The First Affiliated Hospital of Henan University of Traditional Chinese Medicine, Zhengzhou, Henan 450000; ${ }^{2}$ School of Pharmacy, Henan University of Traditional Chinese Medicine, Zhengzhou, Henan 450008;

${ }^{3}$ The Level Three Laboratory of Chinese Traditional Medical Preparation of State Administration of TCM;

${ }^{4}$ Key Laboratory of Viral Diseases Prevention and Treatment of TCM of Henan Province, Zhengzhou, Henan 450000,

P.R. China; ${ }^{5}$ Department of Computer Science and Statistics, University of Rhode Island, Kingston, RI 02881, USA
\end{abstract}

Received May 15, 2016; Accepted July 21, 2016

DOI: $10.3892 / \mathrm{etm} .2016 .3733$

\begin{abstract}
Tools to define the active ingredients and flavors of Traditional Chinese Medicines (TCMs) are limited by long analysis times, complex sample preparation and a lack of multiplexed analysis. The aim of the present study was to optimize and validate an electronic tongue (E-tongue) methodology to analyze the bitterness of TCMs. To test the protocol, 35 different TCM concoctions were measured using an E-tongue, and seven replicate measurements of each sample were taken to evaluate reproducibility and precision. E-tongue sensor information was identified and classified using analysis approaches including least squares support vector machine (LS-SVM), support vector machine (SVM), discriminant analysis (DA) and partial least squares (PLS). A benefit of this analytical protocol was that the analysis of a single sample took $<15 \mathrm{~min}$ for all seven sensors. The results identified that the LS-SVM approach provided the best bitterness classification accuracy (binary classification accuracy, $100 \%$; ternary classification accuracy, 89.66\%). The E-tongue protocol developed showed good reproducibility and high precision within a $6 \mathrm{~h}$ measurement cycle. To the best of our knowledge, this is the first study of an E-tongue being applied to assay the bitterness of TCMs. This approach could be applied in the classification of the taste of TCMs, and serve important roles in other fields, including foods and beverages.
\end{abstract}

Correspondence to: Dr Ruixin Liu, Department of Pharmacy, The First Affiliated Hospital of Henan University of Traditional Chinese Medicine, 19 Renmin Road, Zhengzhou, Henan 450000, P.R. China E-mail: liuruixin7@163.com

*Contributed equally

Key words: methodology, electronic tongue, taste, traditional Chinese medicine

\section{Introduction}

The human gustatory system allows the sense of the following five basic tastes: Sour, bitter, sweet, salty and umami. The biological mechanism of taste involves a series of electrical signals triggered by molecular stimulations of the taste buds. These impulses are conducted to the brain, which interprets these signals as the appropriate taste $(1,2)$.

Historically, tastes were evaluated with human tasterpanels, which suffer from numerous limitations such as tester fatigue, particularly in regards to bitterness. In the early stages of drug development, this method is unsuitable because it has a high cost and is potentially dangerous $(3,4)$. Therefore, analytical taste sensing tools such as the electronic tongue (E-tongue) have been developed to increase safety and reduce costs. E-tongue technology originated from multi-analyte sensing technology between the 1980s and 1990s. The electronic nose was a particularly powerful example of multi-analyte sensing and has been employed in defense and environmental applications (5). Later, researchers extended this to solution-phase analysis for a variety of chemical 'tastes'. Analytes include small molecules, proteins and whole blood cells $(6,7)$. At the core of the technology are multiple sets of taste sensor arrays, whose surface is coated with a 'sensing membrane' material similar to that in biological systems. When a taste substance is adsorbed onto this membrane, data is obtained from the resulting changes in membrane potential. This technology offers an intelligent electronic recognition system, which reflects the overall taste information of a sample (1,8-10).

This taste-sensing technology has been applied to the food industry $(11,12)$ for $>20$ years in numerous roles, including food traceability (13), freshness (14), quality (15-17) and safety inspection $(18,19)$. Increasingly, this technique is being applied in pharmaceutical fields (20-22), where it is frequently used to evaluate the bitterness of medicines and make improvements to their formulations (23-28).

Although the collection of taste information of foods and drugs using the E-tongue, and the following post-hoc processing and analysis, is now fairly common, there remains multiple unresolved issues in regards to precision 
and reproducibility of the results, and the identification of taste between different systems. The present study utilized the E-tongue to collect taste information on medicines that have not been characterized. To the best of our knowledge, this is the first study to optimize the methodology of taste analysis using the E-tongue (22-24). The method established was empirically optimized, validated and applied to collect taste information of a number of TCM decoctions.

\section{Materials and methods}

Apparatus. The present study was performed using an ASTREE II electronic tongue (Alpha M.O.S, Toulouse, France). The E-tongue consisted of a hexadecimal autosampler, a silver/silver chloride reference electrode, a data acquisition system, a workstation running AlphaSoft software (version 12; Alpha M.O.S, Toulouse, France) and seven sensors. The seven sensors were called ZZ2808-2-512 (ZZ), CA2804-2-440 (CA), DA2808-12-330 (DA), BA2808-2-230 (BA), GA2808-2-361 (GA), BB2011-09-141 (BB) and AB2011-10-010 (AB), and were specifically developed for measuring bitterness. The sensors contain two semiconductor regions composed of a thermal insulation material, each covered with a different molecular membrane, with different adsorption properties and the detection thresholds.

Preparation of berberine hydrochloride, rhynchophylline, leonurine, matrine and quinine samples. Samples were weighed using an electronic balance $( \pm 0.1 \mathrm{mg}$ accuracy) at room temperature and dissolved completely in deionized water. Solutions of berberine hydrochloride (lot no. 101002; Sichuan Province Yuxin Pharmaceutical Co., Ltd., Deyang, China), rhynchophylline (lot no. 20100216; Hubei Tungshun Medicine Science \& Technology Co., Ltd., Wuhan, China), leonurine (lot no. SX-091205; Xi'an Hao-Xuan Bio-Tech Co., Ltd., Xi'an, China), matrine (lot no. KS20110725; Xi'an Jiatian Biotechnology Co., Ltd.) and quinine (lot no. 20100510; Shanxi Tianyuan Pharmaceutical Co. Ltd, Yùnchéng, China) were prepared at concentrations of $0,0.025,0.1,0.25,0.5$ and $1 \mathrm{mmol}$ and stored at $4^{\circ} \mathrm{C}$ until required.

Preparation of TCM samples. Ten-fold concentrations of 35 Chinese herbs were prepared, relative to the mean of the prescribed dosage in the Pharmacopoeia of the People's Republic of China. The herbs were placed in 2,000 ml water, soaked for $30 \mathrm{~min}$ and heated in a microwave $(2100 \mathrm{~W})$ until boiling. The power was then reduced to $600 \mathrm{~W}$ and the solution heated for a further $20 \mathrm{~min}$. The remaining herb pieces were filtered out, an additional 2,000 $\mathrm{ml}$ of water was added, the solution heated until boiling and then boiled for $10 \mathrm{~min}$. This process was repeated for each herb. Then, the filtrates of the first and second decoctions were combined, mixed and cooled to room temperature, followed by centrifugation at room temperature for $15 \mathrm{~min}$ at $1,434 \mathrm{x} \mathrm{g}$. The supernatant was collected and the volume adjusted to $4,000 \mathrm{ml}$. Samples were aliquoted, capped, sterilized and stored at $4^{\circ} \mathrm{C}$ until required.

Optimization of measurement time. Purified water was used as the washing solution and $0.5 \mathrm{mmol}$ caffeine solution was used as the sample. The E-tongue was cleaned with washing solution (6 times, $10 \mathrm{sec} / \mathrm{wash}$ ). Then, $80 \mathrm{ml}$ of sample solution was placed in a $120 \mathrm{ml}$ beaker for analysis. Each E-tongue measurement used all 7 sensors, which was taken at room temperature and lasted $120 \mathrm{sec}$. The same sample was measured 10 times consecutively, with the values of last 4 measurements being used to calculate the relative standard deviation (RSD). In addition, RSD was measured in in $10 \mathrm{sec}$ ranges (e.g. 0-9, 1-10 and 2-11 sec).

Optimization of the number of sample measurements. At the beginning of a measurement the signal is unstable, stabilizing as the number of measurements increase. To investigate how many replicates were required for the response signal to become stable, matrine and berberine hydrochloride samples (described previously) were measured using the E-tongue 10 times, with each measurement lasting for $120 \mathrm{sec}$. RSD values were calculated for all measurements and then compared.

Optimization of the order of E-tongue washing and sample measurement. Berberine hydrochloride $(80 \mathrm{ml})$ was added to a $120 \mathrm{ml}$ beaker for E-tongue measurements. The following two measurement schemes were then performed: i) The E-tongue was washed once in-between each measurement of the same sample; and ii) the same sample was measured without washing in-between. In this experiment, the same sample was measured seven times (120 sec each) and the results of last 4 measurements used to calculate the RSD.

Validation of reproducibility. Aqueous solutions of $0.5 \mathrm{mmol}$ rhynchophylline, matrine, quinine and leonurine were prepared in triplicate (12 samples total). E-tongue measurements were conducted under the optimized conditions described above. In this experiment, the same solution was measured seven times (120 sec each) and the results of last 4 measurements used to calculate the RSD. The RSD of each sample prepared in triplicate was then compared to evaluate reproducibility.

Validation of precision within a measurement cycle $(6 \mathrm{~h})$. The measurement time is $<15 \mathrm{~min}$ for a single sample, however, a complete experiment requires the measurement of multiple samples. In order to ensure accuracy of the results, the measurement of all samples need to be finished within a measurement cycle $(6 \mathrm{~h})$. Aqueous solutions of quinine at $0.1,0.5$ and $1.0 \mathrm{mmol}$ were prepared and measured using the E-tongue. Measurements were repeated four times. All measurements were completed within $6 \mathrm{~h}$, and the results of last 4 measurements used to calculate the RSD.

Validation of inter-day precision. Aqueous solutions of quinine at $0.025,0.1,0.25,0.5$ and $1.0 \mathrm{mmol}$ were measured in triplicate on days 1, 2 and 3 to measure E-tongue data reproducibility on different days. In addition, the RSD of E-tongue measurements over the three days was calculated.

Analysis of the taste of TCMs with different degrees of bitterness. In the present study 35 Chinese herbs (lot no. 20110224; Henan Zhongyi Pharmaceutical Co., Ltd., 
Zhengzhou, China) were selected from the 'Pharmacopoeia of the People's Republic of China' (29). This text describes the TCMs used in the present study by the following characteristics: Light smell, tasteless; light smell, slightly bitter taste; light smell, bitter taste; and light smell, very strong bitter taste. The tastes described were evaluated using a human taster panel according to the degree of bitterness (30-32). TCM samples, prepared as described above, were measured using the E-tongue under the optimized conditions described above.

The results of E-tongue measurements and the human taste panel were evaluated using the robust regression analysis method $(33,34)$. E-tongue measurements of 6 samples were excluded, using standardized residuals and score distance as indicators. These findings were in preparation for a publication elsewhere (35). The remaining 29 samples were grouped into two classes or three classes (Table I), based upon their bitterness levels (I-V) described by Liu et al (30). A two-dimensional bitterness classification model was established by considering level I as a class and grouping levels II, III, IV and V as the second class. The three-dimensional classification models consider level I as a class, levels II and II as a second class, and levels IV and V as a third class.

Statistical analysis. Least squares support vector machine (LS-SVM), simple support vector machine (SVM) or discriminant analysis (DA) classification algorithms were used for the classification models. MATLAB (release R2011b; Mathworks, Inc., Natick, MA, USA) and LS-SVMlab Toolbox software [version 1.8; www.esat.kuleuven.be/sisita/lssvmlab (accessed 23/01/16)] was used.

Using the LS-SVM method, the accuracy of the classification models compared with the results of the human taste panel measured, this was then used to select the most appropriate function for study, including linear kernels, polynomial kernels and radial basis functions. For each type of kernel, a self-compiled program screened and optimized the model parameters repeatedly, finally selecting the most appropriate kernel function and parameters. Modeling optimization was performed with SVM (36) and DA (37), with classification accuracy rates of cross-validation calculated separately.

PLS regression analysis was conducted on latent variables. Then, the projection scoring factors in principal component space were used to produce the two- and three-dimensional classification results.

\section{Results and Discussion}

Optimization of measurement time. The aim of the present study was to identify when E-tongue measurements become stable. This is important to assess the validity of any future measurements, as the signal should not be affected by noise. Firstly, sensor measurements of $0.5 \mathrm{mmol}$ caffeine solution showed the characteristic response curve of sensors to the same solution (Fig. 1) and the signal became more stable over time. Fig. 2 shows the RSD values of the seven different sensors to $0.5 \mathrm{mmol}$ caffeine solution. The RSD decreased over time and reached a minimum by $120 \mathrm{sec}$. So, $120 \mathrm{sec}$ measurement times were used for all subsequent measurements.

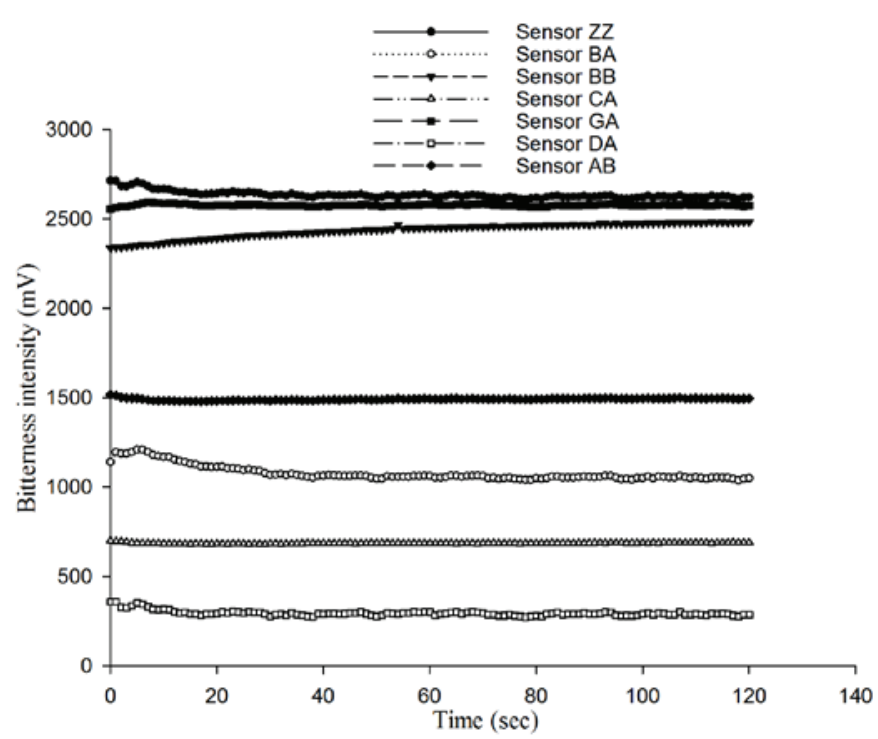

Figure 1. Characteristic response curves of different E-tongue sensors to $0.5 \mathrm{mM}$ caffeine solution.

Optimization of the number of sample measurements taken. Next, the present study investigated how many sample measurements were required to produce a stable response signal. For each test sample, 10 replicate measurements were taken (Fig. 3). RSD was identified to decrease as the number of measurements taken increased. The RSD from taking 4-7 measurements was not reduced further when $>7$ measurements were taken. For example, for the berberine hydrochloride sample, the RSD values of 7 repeats and 10 repeats were 1.89 and 3.05 fold that of 3 repeats (Fig. 3A). For matrine, the RSD values of 4-7 repeats and 7-10 repeats were 1.99 and 2.04 times higher, respectively, than that of 3-6 repeats (Fig. 3B). Therefore, the number of measurements taken of each sample was selected to be 7 . In addition, this will minimize analysis time and extend the E-tongue lifetime.

Determination of the order of E-tongue washing and sample measurements. To determine the best approach to measurements, two different schemes were tested on berberine hydrochloride solutions (Fig. 4). In scheme 1 each measurement of the same sample was followed by a single clean, whereas in scheme 2 measurements of the same sample were taken in a row. Scheme 2 was identified to be more stable because its RSD values were between 1.5 and 2 fold lower compared with those from scheme 1 . The difference between the two schemes, at all respective concentrations, was significant $(\mathrm{P}<0.05$; Fig. 4$)$. In a practical sense, the single cleaning between each measurement used in scheme 1 required the E-tongue to switch back and forth between the sample and its corresponding washing cup. This was more time-consuming than the method used in scheme 2 . Thus, scheme 2 was chosen for the remaining analysis.

Reproducibility of results. The RSD values of the same sample in triplicate were typically $<5 \%$ for the majority of sensors (Fig. 5). However, when the sample was matrine or quinine, the RSD values from sensors DA and GA were $\geq 11 \%$ 

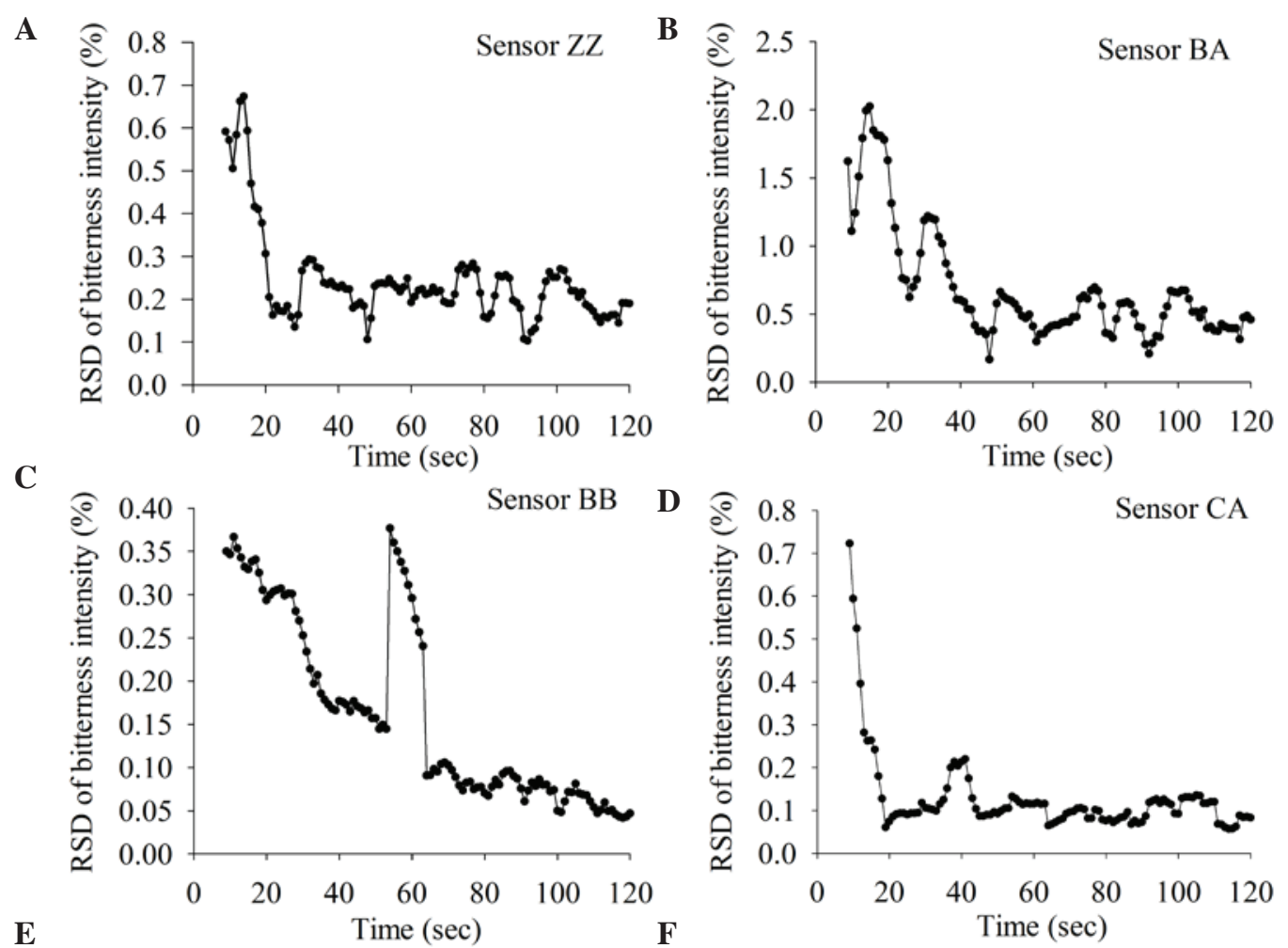

$\mathbf{E}$
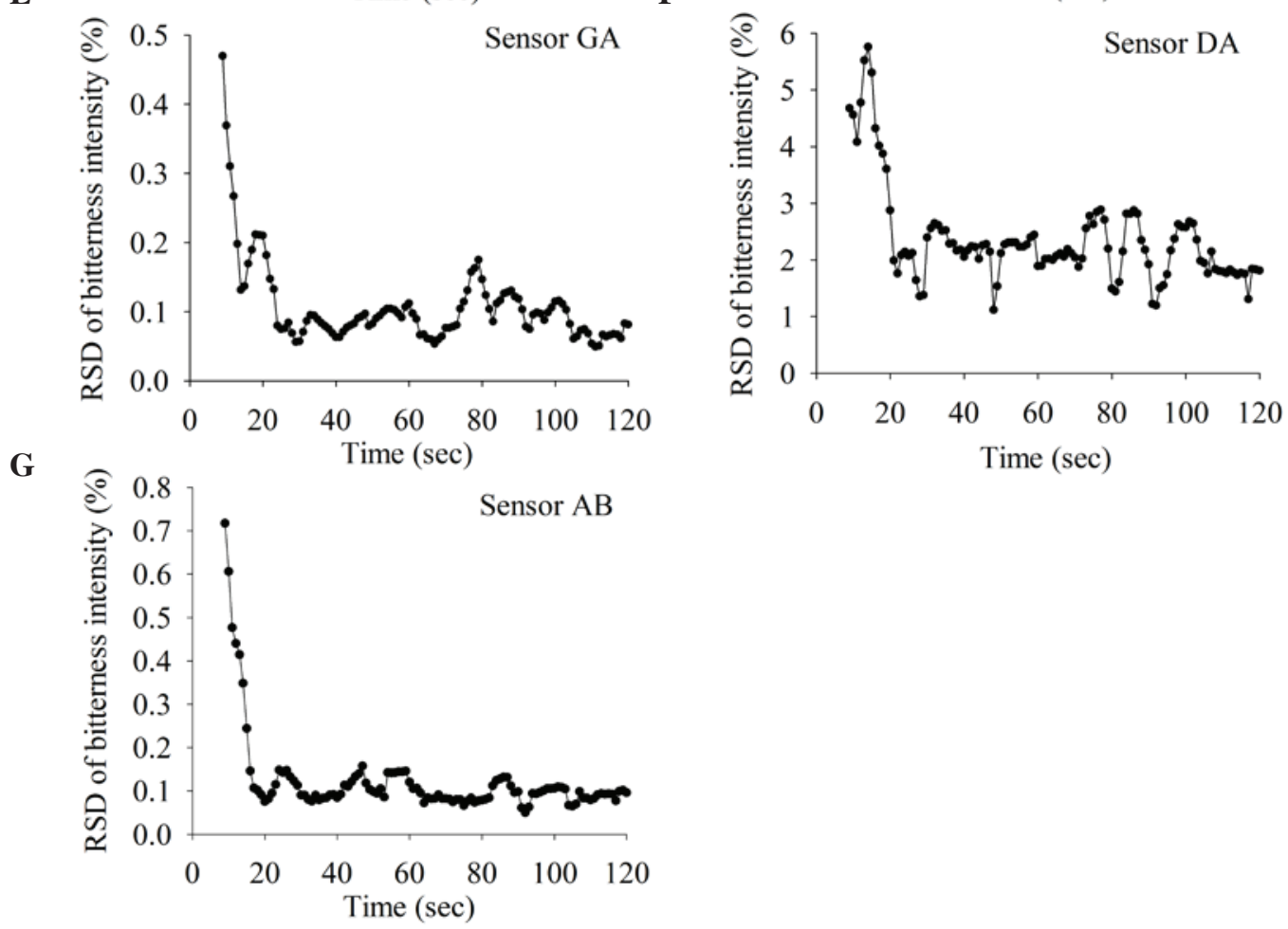

Figure 2. RSD values of the intensity of bitterness of $0.5 \mathrm{mmol}$ caffeine measured by different E-tongue sensors over 120 sec to optimize sample measurement time. The following sensors were tested: (A) ZZ, (B) BA, (C) BB, (D) CA, (E) GA, (F) DA and (G) AB. RSD, relative standard deviation.

(Fig. 5). The baseline responses of all seven sensors were normal and the E-tongue passed its self-checking protocol. Thus, this variation is likely due to the samples and not the E-tongue.

Measurement precision within a $6 \mathrm{~h}$ measurement cycle. Quinine solutions at different concentrations were measured using the E-tongue within a $6 \mathrm{~h}$ measurement cycle (Fig. 6).
The RSD within $6 \mathrm{~h}$ was $<4 \%$, indicating that the approach was suitable for sample analysis.

Inter-day precision of measurements. Quinine solutions of five different concentrations were measured using the E-tongue to evaluate the inter-day precision of measurements (Fig. 7). The results identified that measurements from sensors $\mathrm{ZZ}, \mathrm{BA}, \mathrm{BB}$ and $\mathrm{AB}$ were relatively stable, with an 
A

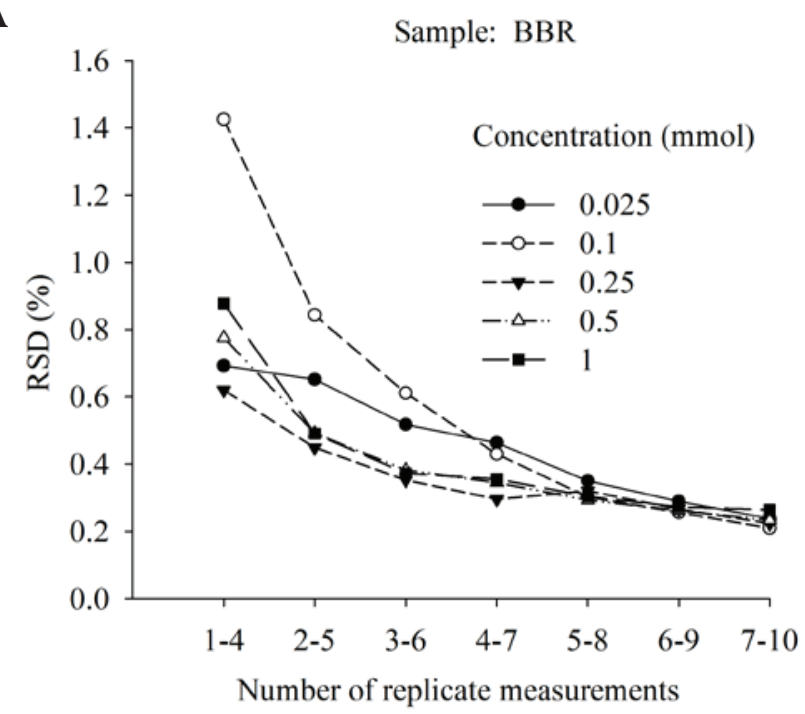

B

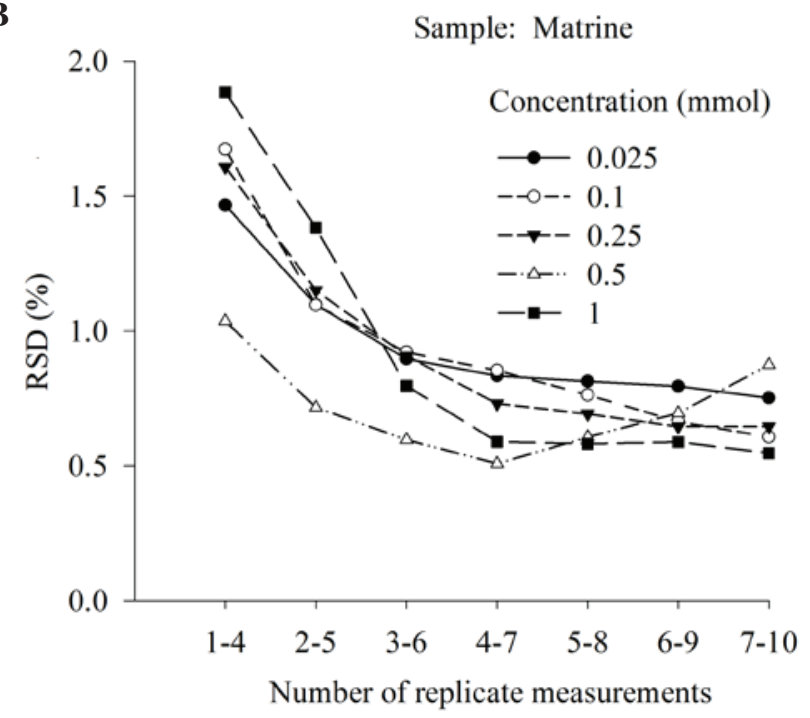

Figure 3. RSD values from E-tongue (all seven sensors) measurements of (A) berberine hydrochloride and (B) matrine solutions with different numbers of replicate measurements. RSD, relative standard deviation.

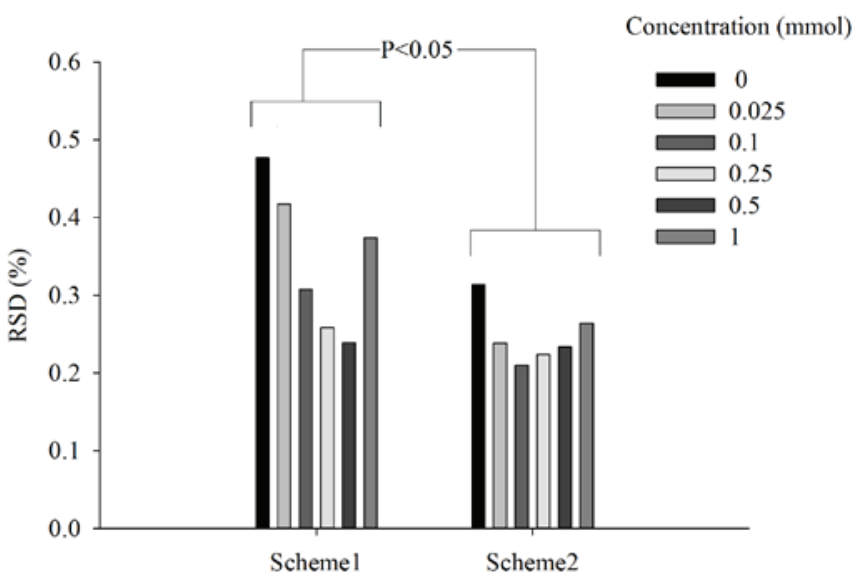

Figure 4. RSD values of berberine hydrochloride solutions by E-tongue (all seven sensors) measurement using two different washing procedures (scheme 1 and scheme 2). RSD, relative standard deviation.

RSD variation over three days of $<10 \%$. Large variations in measurements were found for sensors CA, GA and DA, with RSD values varying by $<37 \%$. Therefore, subsequent measurements were taken on the same day and in the same $6 \mathrm{~h}$ measurement cycle.

Analysis of the bitterness of TCMs. In binary and tertiary classification performed with LS-SVM, the polynomial kernel was selected following optimization. For binary bitterness classification, all 29 samples were correctly classified (human taste panel results), with a cross-validation accuracy rate of $100 \%$. For tertiary bitterness classification, 26 of the 29 samples were correctly classified, with a cross-validation accuracy rate of $89.66 \%$. The misclassified samples were samples 8, 14 and 22 .

In binary and tertiary classification using SVM, the polynomial kernel was selected following optimization. For binary classification, this approach correctly classified 28

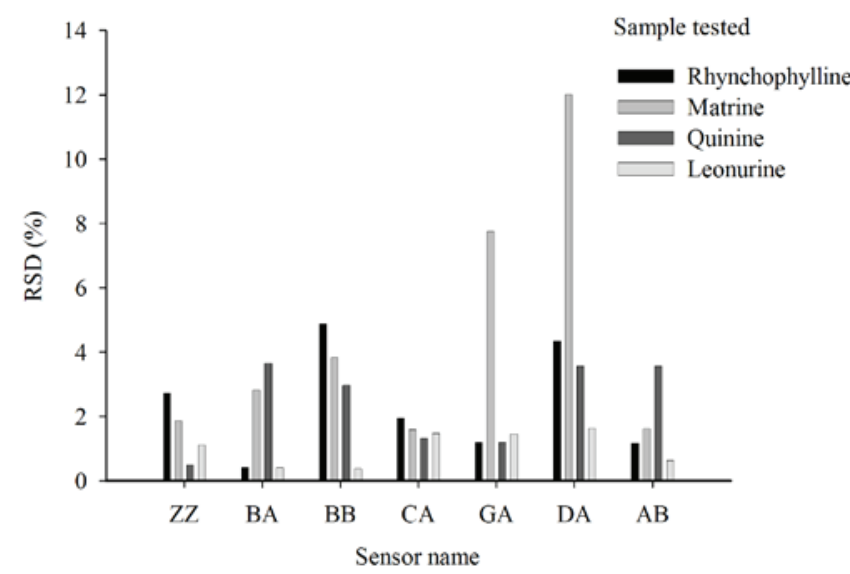

Figure 5. RSD values of different E-tongue sensors to samples measured in triplicate. Results shown are the mean of triplicate measurements. RSD, relative standard deviation.

out of 29 samples, with a cross-validation accuracy rate of $96.55 \%$. Sample 14 was misclassified. For tertiary classification, 25 of the 29 samples were correctly classified with a cross-validation accuracy rate of $86.21 \%$. The misclassified samples were sample 14, 17, 22 and 23.

In binary classification of the 29 samples using DA, 26 out of 29 samples were correctly classified, with a cross-validation accuracy rate of $89.66 \%$. The misclassified samples were 14, 17, and 23. For tertiary classification using DA, 26 out of 29 samples were correctly classified, with a cross-validation accuracy rate of $89.66 \%$. The misclassified samples were sample 14, 17, and 22 .

The results of two-dimensional and three-dimensional bitterness classifications results of 29 samples based on PLS analysis are shown in Fig. 8A and B, respectively. The 29 samples could be grouped into two classes or three classes. However, samples 14 and 17 were on the border of the binary classification and samples 8, 14, 17, 22 and 23 were 
$0.1 \mathrm{mmol}$ quinine

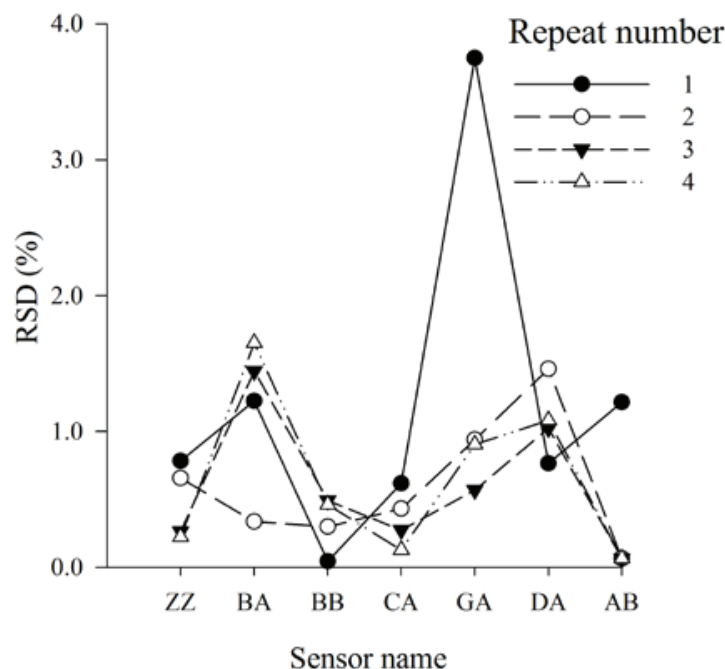

$0.5 \mathrm{mmol}$ quinine

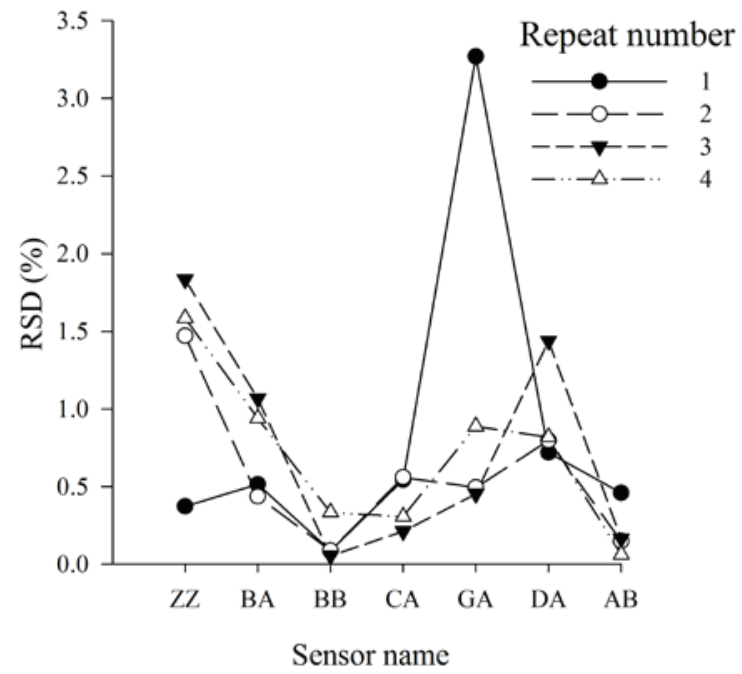

$1 \mathrm{mmol}$ quinine

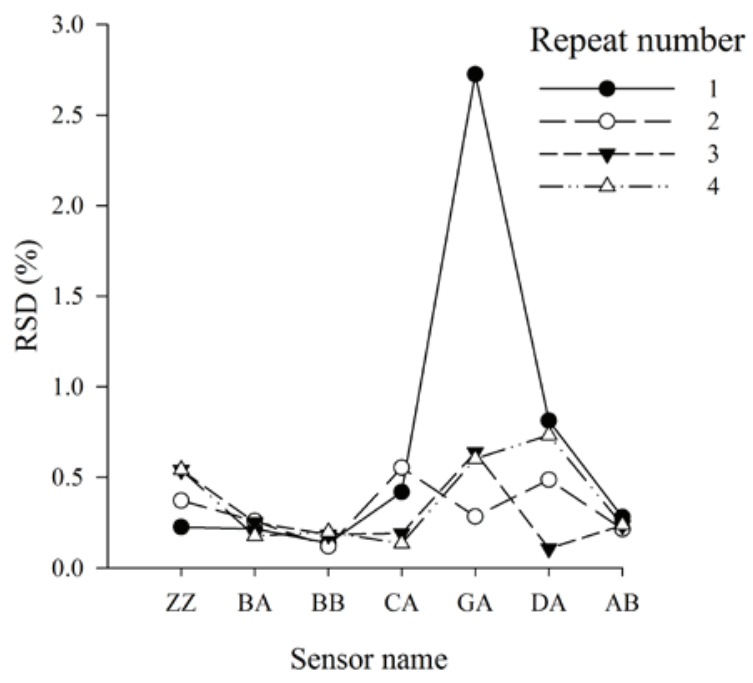

Figure 6. RSD values of different E-tongue sensors to $0.1,0.5$ and $1 \mathrm{mmol}$ quinine solutions within a $6 \mathrm{~h}$ measurement cycle. RSD, relative standard deviation.

on the border of the tertiary classification, so could easily be misclassified.

In conclusion, the present study optimized the E-tongue measurement protocol to use a $120 \mathrm{sec}$ measurement acquisition time, with 7 replicates and optimized the washing process. The optimized washing process comprised of 6 cleans of $10 \mathrm{sec}$ each. The sample solution was then measured continuously, without cleaning in-between measurements of the same sample. Following completion of measurements, the response values of sensors were used for further analysis. This optimized method used had good reproducibility and high precision within $6 \mathrm{~h}$, but poor inter-day precision. Therefore, measurements should be taken within the same $6 \mathrm{~h}$ measurement cycle, rather than on different days or in different measurement cycles.

The optimized protocol was then used to screen and identify the bitterness intensities of 29 TCM decoctions, which were compared to the bitterness intensities previously established by a human taste panel. The results showed that with appropriate data processing, the E-tongue could accurately identify the bitterness intensity of TCM decoctions.

\section{Acknowledgements}

The present study was funded by the following organizations: National Natural Science Foundation for Young Scientists (grant no. 81001646); Special Program for Chinese Medicine Research of Traditional Chinese Medicine Administration of Henan Province (grant no. 2014ZY02066); Fundamental Research Funds for Gifted Young Scientists at Provincial Universities by Henan University of Traditional Chinese Medicine (Zhengzhou, China; grant no. 2014KYYWF-YQ01); and a Collaborative Project with Jiangying Tianjiang Pharmaceutical Co., Ltd. (Jiangyin, 
Table I. TCM samples measured, including the name of the drug and bitterness.

\begin{tabular}{|c|c|c|c|c|c|}
\hline Sample no. & $\begin{array}{l}\text { Chinese } \\
\text { pinyin }\end{array}$ & Drug/sample & Bitterness & $\begin{array}{l}\text { Two-dimensional } \\
\text { classification }\end{array}$ & $\begin{array}{c}\text { Three-dimensional } \\
\text { classification }\end{array}$ \\
\hline 1 & Fuling & Poria & 0.63 & I & $\mathrm{I}$ \\
\hline 2 & Tongcao & Tetrapanacis medulla & 0.64 & $\mathrm{I}$ & $\mathrm{I}$ \\
\hline 3 & Sangzhi & Mori ramulus & 0.67 & $\mathrm{I}$ & $\mathrm{I}$ \\
\hline 4 & Gouteng & Uncariae ramulus cum uncis & 0.70 & $\mathrm{I}$ & $\mathrm{I}$ \\
\hline 5 & Chuanmutong & Clematidis armandii caulis & 0.70 & I & $\mathrm{I}$ \\
\hline 6 & Cheqianzi & Plantaginis semen & 0.71 & I & $\mathrm{I}$ \\
\hline 7 & Mingdangshen & Changii radix & 0.73 & I & I \\
\hline 8 & Tianhuafeng & Trichosanthis radix & 0.91 & I & $\mathrm{I}$ \\
\hline 9 & Zexie & Alismatis rhizoma & 0.95 & I & $\mathrm{I}$ \\
\hline 10 & Zelan & Lycopi herba & 1.19 & $\mathrm{I}$ & I \\
\hline 11 & Cang'erzi & Xanthii fructus & 1.21 & $\mathrm{I}$ & I \\
\hline 12 & Zhuru & Bambusae caulis in taenias & 1.24 & $\mathrm{I}$ & $\mathrm{I}$ \\
\hline 13 & Duzhong & Eucommiae cortex & 1.26 & $\mathrm{I}$ & I \\
\hline 14 & Cheqiancao & Plantaginis herba & 1.26 & I & $\mathrm{I}$ \\
\hline 15 & Baiwei & Cynanchi atrati radix et rhizoma & 1.67 & II & II \\
\hline 16 & Qiancao & Rubiae radix et rhizoma & 1.81 & II & II \\
\hline 17 & Zhebeimu & Fritillariae thunbergii bulbus & 1.82 & II & II \\
\hline 18 & Beidougen & Menispermi rhizoma & 2.03 & II & II \\
\hline 19 & Yanhusuo & Corydalis rhizoma & 2.80 & II & II \\
\hline 20 & Fangji & Stephaniae tetrandrae radix & 3.01 & II & II \\
\hline 21 & Huangqin & Scutellariae radix & 3.28 & II & II \\
\hline 22 & Dangyao & Swertiae herba & 3.92 & II & III \\
\hline 23 & Chuanxinlian & Andrographis herba & 4.04 & II & III \\
\hline 24 & Kumu & Picrasmae ramulus et folium & 4.08 & II & III \\
\hline 25 & Huanglian & Coptidis rhizoma & 4.45 & II & III \\
\hline 26 & Longdan & Gentianae radix et rhizoma & 4.55 & II & III \\
\hline 27 & Huangbo & Phellodendri chinensis cortex & 4.66 & II & III \\
\hline 28 & Huhuanglian & Picrorhizae rhizoma & 4.67 & II & III \\
\hline 29 & Kushen & Sophorae flavescentis radix & 4.78 & II & III \\
\hline
\end{tabular}

Currently, there is no unified bitterness unit. The bitterness score standards used in the present study are described in Liu et al (30).

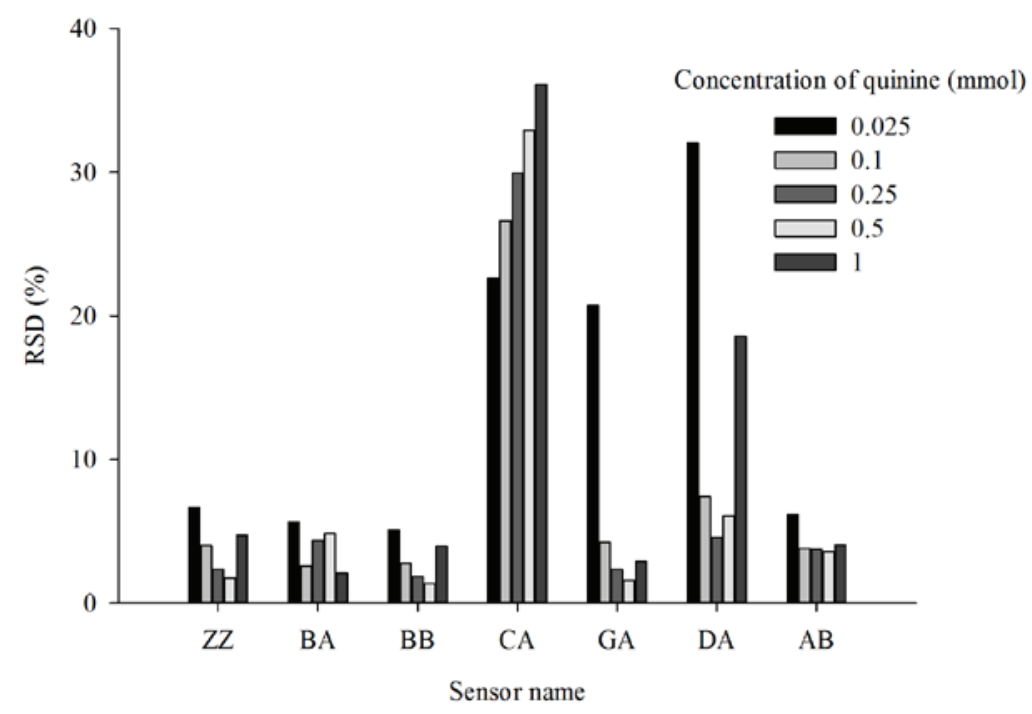

Figure 7. RSD values of E-tongue sensor response to quinine solutions at different concentrations on 3 different days. RSD, relative standard deviation. 
A

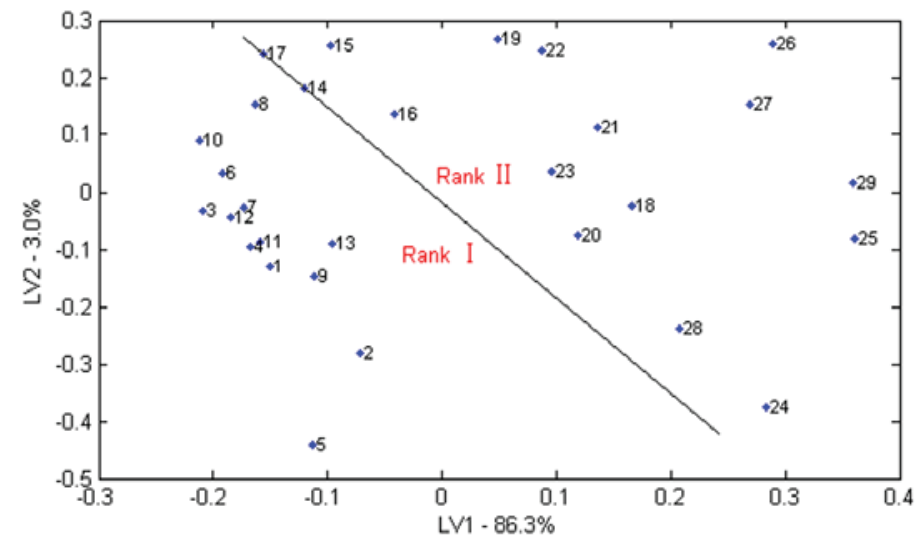

B

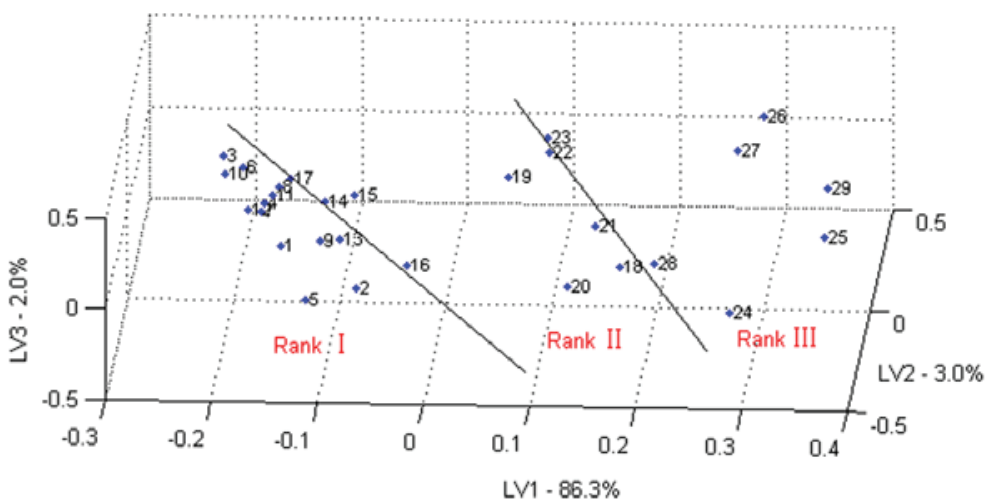

Figure 8. Bitterness classification via PLS of the E-tongue measurements (all seven sensors) of 29 samples. (A) Two-dimensional classification. (B) Three-dimensional classification. Lines represent the divisions between the indicated ranks. LV, latent variable.

China; grant no. XZ2011030042). The authors thank Professor Yuan-Ying Ni and Dr Yang Yang (College of Food Science and Nutritional Engineering, China Agricultural University, Beijing, China) for their guidance and help with the E-tongue.

\section{References}

1. Gupta H, Sharma A, Kumar S and Roy SK: E-tongue: A tool for taste evaluation. Recent Pat Drug Deliv Formul 4: 82-89, 2010.

2. Ayenew Z, Puri V, Kumar L and Bansal AK: Trends in pharmaceutical taste masking technologies: A patent review. Recent Pat Drug Deliv Formul 3: 26-39, 2009.

3. Ito M, Ikehama K, Yoshida K, Haraguchi T, Yoshida M, Wada K and Uchida T: Bitterness prediction of H1-antihistamines and prediction of masking effects of artificial sweeteners using an electronic tongue. Int J Pharm 441: 121-127, 2013.

4. Anand V, Kataria M, Kukkar V, Saharan V and Choudhury PK: The latest trends in the taste assessment of pharmaceuticals. Drug Discov Today 12: 257-265, 2007.

5. Dickinson TA, Michael KL, Kauer JS and Walt DR: Convergent, self-encoded bead sensor arrays in the design of an artificial nose. Anal Chem 71: 2192-2198, 1999.

6. Goodey A, Lavigne JJ, Savoy SM, Rodriguez MD, Curey T, Tsao A, Simmons G, Wright J, Yoo SJ, Sohn Y, et al: Development of multianalyte sensor arrays composed of chemically derivatized polymeric microspheres localized in micromachined cavities. J Am Chem Soc 123: 2559-2570, 2001.

7. Jokerst JV, Jacobson JW, Bhagwandin BD, Floriano PN, Christodoulides N and McDevitt JT: Programmable nano-bio-chip sensors: Analytical meets clinical. Anal Chem 82: 1571-1579, 2010.

8. Riul A Jr, Dantas CA, Miyazaki CM and Oliveira ON Jr: Recent advances in electronic tongues. Analyst 135: 2481-2495, 2010.

9. Baldwin EA, Bai J, Plotto A and Dea S: Electronic noses and tongues: Applications for the food and pharmaceutical industries. Sensors (Basel) 11: 4744-4766, 2011.
10. Toko K, Hara D, Tahara Y, Yasuura M and Ikezaki H: Relationship between the amount of bitter substances adsorbed onto lipid/polymer membrane and the electric response of taste sensors. Sensors (Basel) 14: 16274-16286, 2014.

11. Polshin E, Rudnitskaya A, Kirsanov D, Legin A, Saison D, Delvaux F, Delvaux FR, Nicolaï BM and Lammertyn J: Talanta 81: 88-94, 2010.

12. Peris M and Escuder-Gilabert L: On-line monitoring of food fermentation processes using electronic noses and electronic tongues: A review. Anal Chim Acta 804: 29-36, 2013.

13. Gutiérrez-Capitán M, Santiago JL, Vila-Planas J, Llobera A, Boso S, Gago P, Martínez MC and Jiménez-Jorquera C: Classification and characterization of different white grape juices by using a hybrid electronic tongue. J Agric Food Chem 61: 9325-9332, 2013.

14. Escuder-Gilabert L and Peris M: Review: Highlights in recent applications of electronic tongues in food analysis. Anal Chim Acta 665: 15-25, 2010 .

15. Apetrei C,Apetrei IM, Villanueva S, de Saja JA, Gutierrez-Rosales F and Rodriguez-Mendez ML: Combination of an e-nose, an e-tongue and an e-eye for the characterisation of olive oils with different degree of bitterness. Anal Chim Acta 663: 91-97, 2010.

16. Major N, Marković K, Krpan M, Sarić G, Hruškar M and Vahčić N: Rapid honey characterization and botanical classification by an electronic tongue. Talanta 85: 569-574, 2011.

17. Cetó X, Gutiérrez-Capitán M, Calvo D and del Valle M: Beer classification by means of a potentiometric electronic tongue. Food Chem 141: 2533-2540, 2013.

18. Söderström C, Borén H, Winquist $F$ and Krantz-Rülcker C: Use of an electronic tongue to analyze mold growth in liquid media. Int J Food Microbiol 83: 253-261, 2003.

19. Zhao G, Lin X, Dou W, Tian S, Deng S and Shi J: Use of the smart tongue to monitor mold growth and discriminate between four mold species grown in liquid media. Anal Chim Acta 690: 240-247, 2011.

20. Woertz K, Tissen C, Kleinebudde P and Breitkreutz J: A comparative study on two electronic tongues for pharmaceutical formulation development. J Pharm Biomed Anal 55: 272-281, 2011. 
21. Harada T, Uchida T, Yoshida M, Kobayashi Y, Narazaki R and Ohwaki T: A new method for evaluating the bitterness of medicines in development using a taste sensor and a disintegration testing apparatus. Chem Pharm Bull (Tokyo) 58: $1009-1014,2010$

22. Lorenz JK, Reo JP, Hendl O, Worthington JH and Petrossian VD: Evaluation of a taste sensor instrument (electronic tongue) for use in formulation development. Int J Pharm 367: 65-72, 2009.

23. Zheng JY and Keeney MP: Taste masking analysis in pharmaceutical formulation development using an electronic tongue. Int J Pharm 310: 118-124, 2006.

24. Rachid O, Simons FE, Rawas-Qalaji M and Simons KJ: An electronic tongue: Evaluation of the masking efficacy of sweetening and/or flavoring agents on the bitter taste of epinephrine. AAPS PharmSciTech 11: 550-557, 2010.

25. Tanigake A, Miyanaga Y, Nakamura T, Tsuji E, Matsuyama K, Kunitomo $M$ and Uchida T: The bitterness intensity of clarithromycin evaluated by a taste sensor. Chem Pharm Bull (Tokyo) 51: 1241-1245, 2003.

26. Li L, Naini V and Ahmed SU: Utilization of a modified specialcubic design and an electronic tongue for bitterness masking formulation optimization. J Pharm Sci 96: 2723-2734, 2007.

27. Haraguchi T, Uchida T, Hazekawa M, Yoshida M, Nakashima M, Sanda H, Hase T and Tomoda Y: Ability of food/drink to reduce the bitterness intensity of topiramate as determined by taste sensor analysis. Chem Pharm Bull (Tokyo) 64: 14-20, 2016.

28. Yaroshenko I, Kirsanov D, Kartsova L, Sidorova A, Sun Q Wan H, He Y, Wang P and Legin A: Exploring bitterness of traditional Chinese medicine samples by potentiometric electronic tongue and by capillary electrophoresis and liquid chromatography coupled to UV detection. Talanta 152: 105-111, 2016.
29. Pharmacopoeia of the People's Republic of China 2010. 8th edition, volume I, China Medical Science and Technology Press, Beijing, 2010.

30. Liu R, Zhang X, Zhang L, Gao X, Li H, Shi J and Li X: Bitterness intensity prediction of berberine hydrochloride using an electronic tongue and a GA-BP neural network. Exp Ther Med 7: 1696-1702, 2014.

31. Shi J, Zhang X, Qiu J, Li X and Liu R: Investigation of bitter masking mechanism of $\beta$-cyclodextrin to several traditional Chinese medicines. Zhong Guo Shi Yan Fang Ji Xue Za Zhi 19: 1-4, 2013 (In Chinese).

32. Li X, Wu Z, Liu R, Xu Z, Shi J and Li H: Study on bitterness evaluation of Chinese herbal decoction by THTPM. Zhong Guo Shi Yan Fang Ji Xue Za Zhi 17: 11-13, 2011 (In Chinese).

33. Verboven S and Hubert M: Matlab library LIBRA. Wiley Interdisciplinary Reviews: Computational Statistics 2: 509-515, 2010.

34. Verboven S and Hubert M: LIBRA: A MATLAB library for robust analysis. Chemometr Intell Lab Syst 75: 127-136, 2005.

35. Lin Z, Zhang Q, Liu R, Gao X, Zhang L, Kang B, Shi J, Wu Z, Gui X and Li X: Evaluation of the bitterness of traditional Chinese medicines using an E-tongue coupled with a robust partial least squares regression method. Sensors (Basel) 16: 151, 2016.

36. Newman J, Egan T, Harbourne N, O'Riordan D, Jacquier JC and $\mathrm{O}$ 'Sullivan $\mathrm{M}$ : Correlation of sensory bitterness in dairy protein hydrolysates: Comparison of prediction models built using sensory, chromatographic and electronic tongue data. Talanta 126: 46-53, 2014.

37. Kuligowski J, Pérez-Guaita D and Quintás G: Application of discriminant analysis and cross-validation on proteomics data. Methods Mol Biol 1362: 175-184, 2016. 